\title{
GESTÃO DO CAPITAL INTELECTUAL E DA CAPACIDADE ABSORTIVA COMO FUNDAMENTOS DO DESEMPENHO INOVADOR
}

\author{
MANAGEMENT OF INTELLECTUAL CAPITAL AND ABSORPTIVE CAPACITY AS FUNDAMENTS OF \\ INNOVATIVE PERFORMANCE
}

JÉFERSON DELEON FÁVERO

Doutorando em Ciências Contábeis e Administração - Universidade Regional de Blumenau (FURB)

Professor no Centro Universitário Leonardo da Vinci - UNIASSELVI

Orcid: https://orcid.org/0000-0002-6816-201X / E-mail: jeferson_deleon@ hotmail.com

Endereço: Rua José Woestemeier, 30, Bairro Velha, Blumenau - SC CEP 89-040498

\section{PÉRICLES EWALDO JADER PEREIRA \\ Doutorando em Ciências Contábeis e Administração - Universidade Regional de Blumenau (FURB) \\ Professor no Centro Universitário Leonardo da Vinci - UNIASSELVI \\ Orcid: https://orcid.org/0000-0002-4407-7398 / E-mail: peckjader@gmail.com}

\author{
GIANCARLO GOMES \\ Universidade Regional de Blumenau (FURB) \\ Coordenador do Programa de Pós Graduação em Administração - PPGAd/FURB \\ Doutorado em Ciências Contábeis e Administração - FURB \\ Orcid: http://orcid.org/0000-0003-1174-7161 / E-mail: giancarlog@furb.br \\ LUCIANO CASTRO DE CARVALHO \\ Professor do Programa de Pós Graduação em Administração - PPGAd/FURB \\ Doutor em Administração - FGV/SP \\ Orcid: https://orcid.org/0000-0003-1558-5657 / E-mail: luccar@gmail.com
}

\section{RESUMO}

A cada dia que passa o cenário encontrado nas indústrias se torna mais complexo, e dessa forma existe a necessidade e a busca constante por inovação. O capital intelectual e a capacidade absortiva têm sido observados como um recurso estratégico das organizações devido a capacidade que a empresa possui em criar e aplicar a sua base de conhecimento. Neste contexto, sugere-se que o desempenho inovador (DI) é influenciado pela gestão do capital intelectual $(\mathrm{Cl})$ e da capacidade absortiva (ACAP). Baseado em uma perspectiva mais integradora, o objetivo do artigo é analisar se o desempenho inovador é influenciado pela gestão do (Cl), da (ACAP). Com base em uma amostra de 97 empresas do segmento metalmecânico, utilizou-se da modelagem de equações estruturais para estimar se há influência das dimensões do capital intelectual e da capacidade absortiva nas dimensões do desempenho inovador. Os resultados revelam que tanto o capital intelectual, quanto a capacidade absortiva isoladamente influenciam o desempenho inovador. No entanto, não há evidências de influência quando o capital intelectual e o desempenho inovador são mediados pela capacidade absortiva.

Palavras-chave : Capital intelectual. Capacidade absortiva. Desempenho inovador. 


\begin{abstract}
With each passing day the scenario found in industries becomes more complex, and in this context there is the need and constant search for innovation. Intellectual capital and absorptive capacity have been seen as a strategic resource for organizations because of the company's ability to create and apply its knowledge base. In this context, it is suggested that innovative performance (IP) is influenced by the management of intellectual capital (IC) and absorptive capacity (ACAP). Based on a more integrative perspective, the purpose of the paper is to analyze whether innovative performance is influenced by (ACAP) management. Based on a sample of 97 companies in the metalworking segment, structural equation modeling was used to estimate whether there is influence of the dimensions of intellectual capital and absorptive capacity on the dimensions of innovative performance. The results reveal that both intellectual capital and absorptive capacity alone influence innovative performance. However, there is no evidence of influence when intellectual capital and innovative performance is mediated by absorptive capacity.
\end{abstract}

Keywords: Intellectual capital. Absorptive capacity. Innovative performance.

\title{
1 INTRODUÇÃO:
}

O cenário industrial brasileiro tem se mostrado competitivo e agressivo, visto que o contexto econômico se monstra cada vez mais diversificado e desafiador (FIESC, 2016). Os clientes exigem cada vez mais qualidade, rapidez e benefícios, o que demanda inovação nos processos e nos produtos das organizações, bem como eficiência e eficácia no ambiente industrial. Dessa forma, as características industriais acabam por valorizar os talentos e a quantidade de informações geridas dentro e fora das empresas.

Kim e Shim (2018) colocam que a inovação se mostra como uma vertente do aumento da produtividade e como um fator pressuposto ao crescimento econômico. Isso se apresenta cada vez mais real, frequente e necessário quando as incertezas e os riscos se retratam no ambiente de atuação organizacional (LIAO; CHANG; WU, 2010) e quando se percebe um mercado cada vez mais variável, sazonal e com ciclos de produtos menores (HUNG, 2007).

Kianto, Sáenz e Aramburu (2017) escrevem que a inovação pode ser entendida como a produção, adoção, assimilação e aplicação de algo novo com valor agregado em âmbitos sociais e econômicos, bem como o aperfeiçoamento de serviços, produtos e métodos de gestão. Nesse sentido, tem-se como resultado da inovação, o desempenho inovador (DI), o qual segundo Alegre e Chiva (2013) representa o comportamento inovador das organizações frente as inovações de produtos e as inovações de processo (CHEN et al., 2015).

Em um cenário competitivo como o atual existe a necessidade de se transferir conhecimento externo para dentro das organizações, porém isso não acontece sem algum tipo de custo, pois exige capacidades específicas para que esse procedimento obtenha sucesso. Dessa maneira um aspecto fundamental do processo de criação de conhecimento para inovar emana recursos internos intangíveis, entre eles, o Capital Intelectual $(\mathrm{Cl})$, bem como a capacidade organizacional para absorver conhecimento externo, chamada de Capacidade Absortiva (ACAP).

Nesse sentido, esforços têm sido concentrados em relação ao desenvolvimento de pesquisas acerca do tema. Mariano e Walter (2015) destacam que existe um amplo espaço para o desenvolvimento de pesquisas relacionando o capital intelectual e a capacidade absortiva, principalmente abordando empresas de diferentes portes. Os autores ainda 
defendem que pesquisas empíricas abordando os constructos do capital intelectual e da capacidade absortiva devem fornecer contribuições gerenciais para as organizações.

Jordão e Novas (2017) destacam ainda que a inovação é fundamental para aumentar as chances de sobrevivência das empresas, e que isso acontece na medida que as organizações aproveitam as oportunidades e sustentam suas vantagens competitivas. Defende-se nesse estudo que os ativos intangíveis (capital intelectual) podem influenciar a capacidade absortiva das empresas, tornando-as mais efetivas em seu desempenho inovador.

Assim sendo, optou-se por investigar o capital intelectual e a capacidade absortiva no setor metalmecânico, uma vez que neste ambiente tem-se alta competitividade, forte influência tecnológica, com abrangência nacional e internacional, além de ser uma das áreas mais dinâmicas da economia. Logo, toda incerteza, instabilidade, dinamismo e complexidade do setor demanda que as organizações absorvem do mercado informações, processos e inovações (FIESC, 2016).

Seguindo nesse contexto, o estudo apresenta a seguinte questão problema: Qual a relação entre a gestão do capital intelectual e da capacidade absortiva no desempenho inovador? Para auxiliar na questão problema, elaborou-se o objetivo do estudo correspondente que será o de analisar a relação entre a gestão do capital intelectual e da capacidade absortiva no desempenho inovador.

Ao apoiar a defesa de mais pesquisas acerca do tema abordado Soo et al. (2017), Machado et al. (2017) e Mariano e Walter (2015) indicam a necessidade de aprofundar estudos envolvendo capital intelectual e capacidade absortiva, o que traz a prerrogativa de analisar se estes dois aspectos apresentam relação no desempenho inovador nas indústrias do segmento metalmecânico.

\section{APORTE TEÓRICO}

\subsection{CAPITAL INTELECTUAL (Cl)}

O estudo sobre a gestão do conhecimento nas organizações tem aberto caminhos para investigações do Capital Intelectual - $\mathrm{Cl}$. Esta abertura propiciou pesquisas sobre o tema Cl por Sveiby (1997) e Edvinsson e Malone (1998), os quais abordaram inicialmente ambientes institucionais dentro das organizações com foco na valoração de ativos intangíveis de forma monetária e não monetária (VAZ; SELIG; VIEGAS, 2019; CASSOL et al., 2016).

Branco e Delgado (2011) e Mcphail (2009) indicam que inicialmente o objetivo de medir o $\mathrm{Cl}$ visava entender os elementos do ambiente contábil como forma de mensurar os recursos e as capacidades intangíveis e tangíveis das organizações em relatórios, tendo como foco os retornos financeiros (VAZ; SELIG; VIEGAS, 2019).

Em um contraponto, Stewart (1998) salienta que as organizações ainda consideram o poder e a importância dos ativos tangíveis como fatores de análises contábeis. Kaplan e Norton (1997) e Stewart (1998) entendem que isso acontece mesmo sabendo que a presença da informação e do conhecimento estão indicando um novo modelo de condução nas empresas, e que, o valor encontrado nas habilidades dos indivíduos e na capacidade de aprendizagem, como ativos intangíveis são o que realmente trarão os resultados esperados pelas organizações.

E nesse sentido, lenciu e Matis (2011) e Stewart (1998) destacam o $\mathrm{Cl}$ como um ativo valioso, permitindo que a organização ganhe robustez competitiva no ambiente onde atua, uma vez que o $\mathrm{Cl}$ é considerado a somatória do conhecimento de todos indivíduos dentro de uma empresa (VAZ; SELIG; VIEGAS, 2019; GRACIOLI et al., 2012). 
Além desta, há diversas definições de $\mathrm{Cl}$ na literatura que são convergentes a lenciu e Matis (2011) e Stewart (1998) como as obras de Davenport e Prusak (1998), Edvinsson e Malone (1998) e Nahapiet e Ghoshal (1998), bem como pesquisas que apresentam convergências parciais, entre eles, Giuliane (2009) e Sveiby (1998).

Salienta-se ainda, que Giuliane (2009) e Sveiby (1998) utilizaram principalmente, da teoria do $\mathrm{Cl}$ para construção de trabalhos com vieses contábeis e com nomenclaturas diferentes, porém com sentido semelhante, destacando o indivíduo, a estrutura e o cliente, conforme também mencionado por Vaz, Selig e Viegas (2019) e Jordão e Novas (2017).

O Capital Humano (CH) representa todos os recursos humanos envolvidos em um processo (funcionários, clientes, fornecedores, investidores). Becker (1962) em seu estudo seminal, considera o $\mathrm{CH}$ como um conjunto de capacidades e de competências individuais, de não propriedade da organização, mas que refletem no desempenho da empresa quando utilizado de forma conjunta.

Segundo lenciu e Matis (2011) e Bontis (1999) o CH é fonte de renovação estratégica e de inovação, com destaque para a pesquisa, desenvolvimento de novos processos e melhoria das competências pessoais.

Edvinsson e Malone (1998) referem-se ao $\mathrm{CH}$ como a capacidade, conhecimento, poder de inovação, habilidade, criatividade e experiências individuais. Para Jordão e Novas (2017) e Cricelli et al. (2014) esses aspectos podem ser transformados em produtos e serviços, o que traz um diferencial para as organizações, bem como atesta a opção de escolha de uma empresa em detrimento de outra.

Tanto Edvinsson e Malone (1998) como Jordão e Novas (2017) entendem que a valorização do $\mathrm{CH}$ significa perceber as pessoas como elementos essenciais, e destacam a importância desse capital para as organizações. E, utilizando-o de forma eficiente, o $\mathrm{CH}$ é considerado um recurso fundamental na estratégia de inovação, uma vez que apenas o ser humano tem a capacidade de absorver, assimilar e transformar a informação em conhecimento, a fim de utilizá-lo (JORDÃO; NOVAS, 2017).

O segundo elemento, considerado capital estrutural (CE), inclui a cultura organizacional, o capital de infraestrutura e os processos (JORDÃO; NOVAZ, 2017; IENCIU; MATIS, 2011). Stewart (1998) entende que o CE, diferente do $\mathrm{CH}$, é de propriedade da empresa, uma vez que o conhecimento institucionalizado e a experiência codificada residente nas empresas permanecem nela, independente da ausência do indivíduo. Como exemplo de CE, Cabrita (2009) e Youndt, Subramaniam e Snell (2004) citam os bancos de dados, as marcas e patentes, a propriedade intelectual, os manuais, as estruturas, os sistemas, as rotinas e os processos organizacionais.

Salienta-se que o CE também é constituído pelos processos e procedimentos de inputs intelectuais (Carson et al., 2004), que por sua vez utilizam de ferramentas que a organização fornece, com o intuito de reter e transferir o conhecimento ao longo das atividades de negócio (VAZ; SELIG; VIEGAS, 2019; IENCIU; MATIS, 2011; CABRITA; BONTIS, 2008).

Para lenciu e Matis (2011) o CE destaca-se também pela arquitetura disponível, onde utiliza-se de sua infraestrutura, instrumentos gerenciais e de seus sistemas de informação para inovar e desenvolver novos produtos e serviços. Essas ações estão condicionadas a atender as demandas mercadológicas, aumentar seu marktshare, produzir com mais eficiência, reduzindo os custos e as perdas (JORDÃO; NOVAS, 2017, KIANTO; SÁENZ; ARAMBURU, 2017; GRACIOLI et al., 2012). 
O capital relacional (CR) segundo lenciu e Matis (2011) refere-se as conexões que a organização possui com seus clientes, fornecedores, colaboradores e stakeholders. Ou seja, é a ligação que a empresa possui com o mundo exterior e consigo mesmo (CABRITA, 2009; BONTIS, 1999). O CR é arguido por Subramaniam e Yondt (2005) como o conhecimento criado internamente e de forma coletiva na organização, por meio de grupos de trabalhos, grupos de melhorias e pelas relações entre os colaboradores.

Stewart (1998) afirma que o CR é o capital mais valioso de todos os ativos intangíveis da organização. As considerações de Edvinsson e Malone (1998) e Vaz e Selig (2016) convergem com Stewart (1998) ao defenderem que as empresas devem promover o bom relacionamento tanto com seus colaboradores, quanto com os stakeholders, visando uma política de melhoria contínua para atender as necessidades dos seus clientes, em sua organização e também fora dela (SUBRAMANIAM; YONDT, 2005). Para Cassol et al. (2016) toda essa proximidade no relacionamento fomenta a melhoria e a inovação em processos, em produtos e na gestão organizacional.

Nessas condições, elaborou-se as seguintes hipóteses a serem testadas: $H 1$ :: $O$ capital intelectual influencia positivamente no desempenho inovador; e, H2.: O capital intelectual influencia positivamente na capacidade absortiva;

\subsection{CAPACIDADE ABSORTIVA (ACAP)}

A capacidade absortiva (ACAP) pode ser entendida como a capacidade da organização em adquirir e assimilar um conhecimento externo, para transformá-lo e modificálo em um novo conhecimento. Esse processo, por sua vez, tem o objetivo de utilizar o novo conhecimento, e consequentemente trazer retorno financeiro e/ou social à organização (NAZARPOORI, 2017; MORÉ et al., 2014; TODORVA; DURISIN, 2007; ZAHRA; GEORGE, 2002).

De forma semelhante, Curado et al. (2017) e Cassol et al. (2016) configuram a ACAP como a habilidade da organização de avaliar o valor dos novos conhecimentos, bem como assimilar essas informações para que sejam aplicadas na empresa, a fim de buscar vantagem competitiva.

Contudo, foi a partir do trabalho de Cohen e Levinthal (1990) que o conceito da ACAP (Absorptive Capacity) começou a ser aplicado no contexto das organizações, em nível de análise organizacional. A ênfase dos autores foi colocada no papel do ambiente externo na determinação da ACAP de uma empresa (FERREIRA; FERREIRA, 2017).

Sob a abordagem organizacional e processual, a ACAP teve sua origem teórica baseada nas dimensões da capacidade dinâmica (ZOLLO; WINTER, 2002; TEECE; PISANO; SHUEN, 1997), da aprendizagem organizacional (EASTERBY-SMITH, 1997), e foi refinada com a teoria da gestão do conhecimento (CHIVA, ALLEGRE, 2005). Além disso, sua essência, referese à capacidade da organização buscar informações e transformá-las em conhecimento aplicável.

Cohen e Levinthal $(1989,1990)$ sugerem que, o desenvolvimento da ACAP de uma organização é baseado em investimentos nas capacidades de absorção dos seus membros. Incrementam ainda, que esta capacidade tende a ser desenvolvida cumulativamente, por ser dependente da trajetória e da capacidade da organização para compartilhar conhecimento e comunicá-lo internamente.

Após várias pesquisas desenvolvidas por Cohen e Levinthal (1990), Zahra e George (2002) reconceituaram o constructo ACAP definindo-a como "um conjunto de rotinas organizacionais e processos pelos quais as organizações adquirem, assimilam, transformam e aplicam o conhecimento para produzir uma organização dinâmica" (ZAHRA; GEORGE, 2002, 
p. 185). Nesta perspectiva a ACAP é um constructo multidimensional formado por rotinas e processos organizacionais.

No modelo de Zahra e George (2002) a ACAP é dividida em dois componentes: O primeiro é a capacidade absortiva potencial (PACAP) que envolve a aquisição, isto é, a capacidade de uma empresa identificar e adquirir conhecimentos gerados externamente que seja fundamental às suas operações, bem como a assimilação, que envolve as rotinas e os processos que permitem analisar, interpretar e compreender a informação adquirida.

O segundo componente é a capacidade absortiva realizada (RACAP), que envolve a transformação e a aplicação. Na transformação, o conhecimento adquirido e assimilado no componente anterior, é aperfeiçoado para combinar os novos conhecimentos com os já existentes na organização. Na etapa da aplicação o conhecimento é estendido, a fim de gerar novas competências organizacionais.

Moré et al. (2014) contribui com o conceito da ACAP ao ressaltar que a capacidade absortiva de uma organização dependerá da capacidade de aquisição, da aprendizagem de seus membros e da forma como essas capacidades serão operadas para obtenção da eficiência e da eficácia organizacional.

Ademais, Moré et al. (2014) salientam que dependerá da capacidade de buscar e reter conhecimentos externos, de transformar, readequar e criar conhecimentos internamente, bem como aplicá-los em produtos e serviços, a fim de alcançar um desempenho inovador. Desta forma, torna-se possível a formulação da hipótese H3.: A capacidade absortiva influencia positivamente no desempenho inovador.

\subsection{DESEMPENHO INOVADOR (DI)}

Inovação segundo Jiménez-Jiménez e Sanz-Valle (2011) é entendida como a exploração e a transformação do conhecimento dentro e fora das organizações por seus colaboradores, principalmente quando compartilhada. Para Gunday et al. (2011) e Alegre e Chiva (2008) a inovação tem sido preponderante para determinar e organizar as estratégias das empresas, tem servido como mecanismo para aumentar a participação no mercado, bem como para mantê-las vivas frente a competitividade presente, independente do segmento de atuação.

Segundo Zhuang, Wiliamson e Carter (1999) esse cenário competitivo tem motivado as organizações a buscarem vantagem utilizando a inovação de produtos e processos. A inovação no processo organizacional envolve atividades e elementos voltados a processos em operações, como tarefas, distribuição de produtos, prestação de serviços, dentre outros (KIM; SHIM, 2018; JONASH; SOMMERLATTE, 2001).

No que tange aos produtos inovadores, Jonash e Sommerlatte (2001) definem como sendo produtos desenvolvidos e criados para atender as demandas expostas pelo consumidor, e que satisfaça consequentemente, suas necessidades.

No âmbito das pequenas e médias empresas, maior percentual amostral desta pesquisa, Rosenbusch, Brinckmann e Bausch (2011) citam que a inovação tem um efeito positivo em relação ao desempenho. Contudo, tal cenário é influenciado pelo tipo de inovação, dependerá da maturidade da empresa e do ambiente cultural que a organização está inserida (KIM; SHIM, 2018; NAZARPOORI, 2017).

Nesta investigação, segmentada ao eixo metalmecânico e que envolve a inovação de produtos e de processos, serão abordadas duas diferentes variáveis: Eficácia e eficiência, as quais são pertencentes a dimensão Desempenho Inovador (DI) (NAZARPOORI, 2017; ALEGRE; LAPIEDRA; CHIVA, 2006). 
Por meio de diversas pesquisas Alegre, Lapiedra e Chiva (2006) desenvolveram uma escala de medida de desempenho em inovação de produtos intitulado como Desempenho Inovador (DI). Os primeiros estudos dos autores foram realizados em empresas vinculadas a biotecnologia. Posteriormente, estudos empíricos de Henttonen, Ritala e Jauhiainen (2011), Moreno, Realy e Rosa (2011) e Bakar e Ahmad (2010) ampliaram esse modelo para outros cenários e segmentos.

Segundo Henttonen, Ritala e Jauhiainen (2011), Bakar e Ahmad (2010) e Alegre, Lapiedra e Chiva (2006) a eficácia tem o objetivo de verificar como a inovação impacta economicamente na empresa, refletindo assim o seu nível de sucesso. Ou seja, a eficácia busca entender qual é o resultado da inovação na organização.

Quanto a eficiência da inovação, refere-se a como os recursos das organizações são consumidos para atingir determinado resultado (BROWN; EISENHARDT, 1995). Alegre, Lapiedra e Chiva (2006) ratificam que a eficiência da inovação se refere a forma que o esforço é realizado para alcançar determinado objetivo.

Nesta consoante, Wheelwright e Clark (1992) indicam que o custo e o tempo são vertentes determinantes para mensurar a eficiência da inovação de produto na elaboração de um projeto de inovação. Postula-se ainda, que o custo e o tempo são medidos por métricas objetivas e subjetivas, conforme também ressaltado por Alegre, Lapiedra e Chiva (2006).

Desta forma, torna-se possível a formulação da hipótese H4.: A capacidade absortiva media a relação entre capital intelectual e o desempenho inovador.

\section{DELINEAMENTO METOdOLÓGICO}

O método utilizado nesta pesquisa fundamenta-se como descritivo, confirmatório e quantitativo, devido ao tema, uma vez que a literatura apresenta poucos estudos frente ao Desempenho Inovador (DI) sob a mediação da capacidade que a indústria tem em absorver informações e mecanismos do ambiente externo.

Por se apresentar em segmento único, esta investigação possibilita que a análise demonstre os diversos movimentos das indústrias diante de seu desempenho inovador. $\mathrm{O}$ setor metalmecânico brasileiro, conforme a Federação da Indústria do Estado de Santa Catarina (FIESC, 2016) tem destaque no cenário mundial. O estado catarinense, inclusive a região do Vale do Itajaí tem contribuído como um grande gerador de empregos, tendo como característica o predomínio de empresas de micro e pequeno porte, ao lado de grandes empresas líderes no setor.

O questionário foi composto de 61 questões, sendo dividido em três etapas: 1 - Capital Intelectual; 2 - Capacidade Absortiva; e, 3 - Desempenho Inovador, conforme Quadro 1.

Quadro 1 - Composição estrutural do questionário de pesquisa

\begin{tabular}{|c|c|c|c|c|}
\hline Dimensão & $\begin{array}{c}\text { Formação da } \\
\text { dimensão }\end{array}$ & Questões & AFC & Principais autores pesquisados \\
\hline \multirow{3}{*}{$\begin{array}{l}\text { Capital } \\
\text { Intelectual }\end{array}$} & Humano & 9 & 5 & \multirow{3}{*}{$\begin{array}{l}\text { Vaz, Selig e Viegas (2019); Jordão e Novas (2017); } \\
\text { Gracioli et al. (2012); Cabrita e Bontis (2008); Carson } \\
\text { et al. (2004). }\end{array}$} \\
\hline & Estrutural & 9 & 4 & \\
\hline & Relacional & 9 & 4 & \\
\hline \multirow{4}{*}{$\begin{array}{l}\text { Capacidade } \\
\text { Absortiva }\end{array}$} & Aquisição & 5 & 4 & \multirow{4}{*}{$\begin{array}{l}\text { Nazarpoori, (2017); Cassol et. al. (2016); Todorova e } \\
\text { Durisin (2007); Zahra e George (2002); Cohen e } \\
\text { Levinthal (1990). }\end{array}$} \\
\hline & Assimilação & 5 & 5 & \\
\hline & Transformação & 6 & 5 & \\
\hline & Aplicação & 4 & 4 & \\
\hline \multirow{2}{*}{$\begin{array}{l}\text { Desempenho } \\
\text { Inovador }\end{array}$} & Eficácia & 10 & 5 & \multirow{3}{*}{$\begin{array}{l}\text { Kim e Shim (2018); Nazarpoori (2017); Henttonen, } \\
\text { Ritala e Jauhiainen (2011), Bakar e Ahmad (2010) e } \\
\text { Alegre, Lapiedra e Chiva (2006). }\end{array}$} \\
\hline & Eficiência & 4 & 3 & \\
\hline \multicolumn{2}{|c|}{ Total de questões } & 61 & 39 & \\
\hline
\end{tabular}

Fonte: Dados da pesquisa. 
O trabalho foi construído e medido por meio da escala Likert com pontuações variando de 1 a 7, sendo 1 para "Discordo plenamente" e 7 "Concordo plenamente". A população pesquisada corresponde a 416 indústrias do segmento metalmecânico da região do Vale do Itajaí, SC, Brasil (FIESC, 2016). Obteve-se como amostra 97 empresas respondentes das 390 solicitações. Dos 97 respondentes, validou-se 96 questionários. Salienta-se que foi realizado análise unidimensional devido a 2 itens faltantes nas respostas, os quais foram preenchidos por meio da média da dimensão. Depois realizou-se a análise de outliers, sendo que nesta, foi retirado do questionário 1 respondente. Foi solicitado uma resposta por empresa de pessoas que estão diretamente ligadas ao desenvolvimento de processos e ao desempenho dos novos produtos (gerentes de projetos, de P\&D, industrial, de marketing, de vendas, comercial, diretores e proprietários), conforme demonstrado no Quadro 2.

Quadro 2 - Características das empresas respondentes

\begin{tabular}{|l|l|c|}
\hline \multicolumn{1}{|c|}{ Porte da empresa } & \multicolumn{1}{|c|}{ Cargo dos respondentes } & $\begin{array}{c}\text { Empresas } \\
\text { respondentes }\end{array}$ \\
\hline \multirow{4}{*}{$\begin{array}{l}\text { Micro e Pequeno (até } 300 \\
\text { colaboradores) }\end{array}$} & $\begin{array}{l}\text { Setor de P\&D } \\
\text { Gerente industrial, proprietário, } \\
\text { diretor }\end{array}$ & 13 \\
\cline { 2 - 3 } & $\begin{array}{l}\text { Setor de vendas marketing, } \\
\text { comercial }\end{array}$ & 14 \\
\cline { 2 - 3 } & Setor de projetos & 21 \\
\hline \multirow{2}{*}{$\begin{array}{l}\text { Médio (de 301 a 500 } \\
\text { colaboradores) }\end{array}$} & Setor de projetos & 1 \\
\hline \multirow{4}{*}{$\begin{array}{l}\text { Grande (acima de } 500 \\
\text { colaboradores) }\end{array}$} & Setor de P\&D & 4 \\
\cline { 2 - 3 } & $\begin{array}{l}\text { Gerente industrial, proprietário, } \\
\text { diretor }\end{array}$ & 1 \\
\cline { 2 - 3 } & $\begin{array}{l}\text { Setor de vendas marketing, } \\
\text { comercial }\end{array}$ & 1 \\
\cline { 2 - 3 } & Setor de projetos & 1 \\
\hline
\end{tabular}

Fonte: Dados da pesquisa.

As organizações deste segmento foram divididas em micro e pequenas; médias; e grandes, conforme o número de empregados apresentados no Quadro 2, segundo critérios da FIESC (2016).

No estudo foi identificada 4 hipóteses denominadas entre $\mathrm{H} 1$ e $\mathrm{H} 4$, as quais possibilitam explicações e testes de ideias relacionadas aos fenômenos propostos, além de orientar os pesquisadores na condução do estudo. Além disso, a investigação caracteriza-se como corte transversal (HAIR JR. et al., 2005), considerando que a coleta se deu em apenas um período, aplicado entre 9 de novembro de 2018 e 20 de janeiro de 2019.

A análise dos dados, foi realizado por meio da técnica de Modelagem de Equações Estruturais (MEE). Em seguida realizou-se a Análise Fatorial Confirmatória (AFC), a confiabilidade dos constructos por meio da Carga Fatorial, $\mathrm{R}^{2}$, Variance Inflation Factor (VIF), $P$-value (abaixo de 0,10), Confiabilidade Composta, Alpha de Cronbach e Variância Média Extraída. Após esta primeira avaliação foram feitas as análises descritivas da amostra, e na sequência apropriou-se novamente da técnica de MEE para testar as relações entre os constructos da pesquisa, conforme desenho apresentado na Figura 1. 
Figura 1 - Desenho de pesquisa

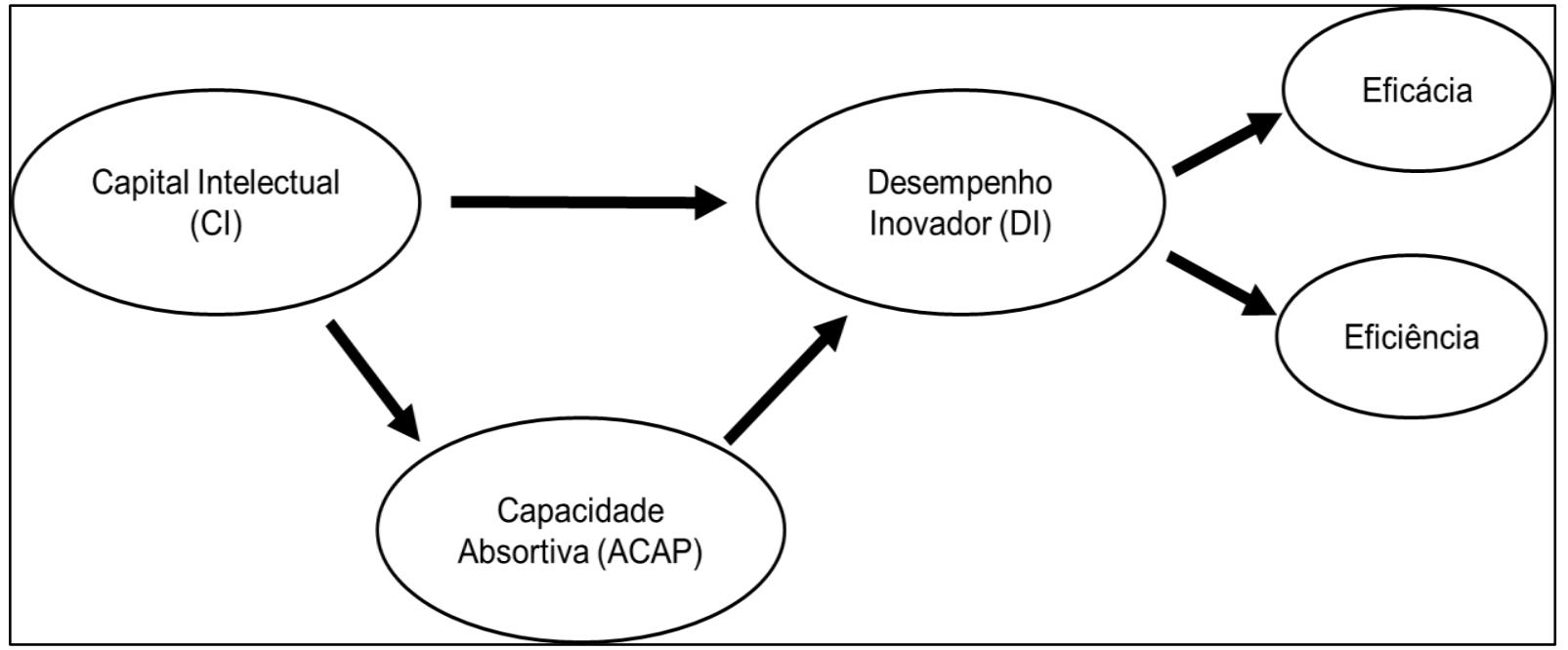

Fonte: Elaborado pelos autores.

A Modelagem de Equações Estruturais apresenta-se como uma técnica multivariada que combina aspectos de regressão múltipla e de análise fatorial, que estimam relações de dependência inter-relacionadas de maneira simultânea (HAIR JR; RINGLE; SARSTEDT, 2013).

A análise dos dados foi conduzida por meio do software SmartPLS, uma vez que este mecanismo permite realizar análises com amostras entre 30 a 100 casos (CHIN; NEWSTED, 1999).

\section{RESULTADOS}

\subsection{MENSURAÇÃO DO MODELO INICIAL}

Com o objetivo de medir a relação das variáveis latentes e as manifestas associadas às variáveis observadas utilizou-se neste primeiro momento da análise o método de estimação do modelo de mensuração inicial. Com a Análise Fatorial Confirmatória (AFC) checou-se os $\beta$ dos caminhos entre as variáveis latentes e as manifestas, com o objetivo de purificar o modelo.

Ao realizar a AFC permaneceram na base de cálculo somente os valores $\beta$ maiores que 0,7 , conforme sugerem Hair Jr, Ringle, Sarstedt (2013). Após os testes foram retiradas 22 variáveis, sendo 14 do constructo $\mathrm{Cl}, 2$ variáveis do constructo ACAP e 6 do DI, obtendo assim, a estrutura de análise final, representado pela Figura 2.

Com o objetivo de identificar a multicolinearidade nos indicadores, utilizou-se o valor da VIF (Variance Inflaction Factor). Esse teste permite verificar se as variáveis independentes diferentes possuem relações lineares exatas ou próximas. Hair Jr, Ringle, Sarstedt (2013) recomendam que o valor da VIF seja menor do que 5 para que a multicolinearidade não seja considerada severa. Constatou-se na análise da multicolinearidade que 2 variáveis da dimensão ACAP, 5 do $\mathrm{Cl}$ e 1 variável do DI (eficácia) se mostraram com valor acima de 5 e foram excluídas da análise.

Com o objetivo de checar a consistência interna do modelo, verificou-se os valores da confiabilidade composta (CC) e do Alfa de Cronbach de cada dimensão. Hair Jr, Ringle, Sarstedt (2013) sugerem que os valores da CC e do Alfa de Cronbach das dimensões sejam maiores que 0,7 , o que por sua vez foi percebido na mensuração inicial em todas as dimensões. Ainda, ressalta-se que a CC tem sido declarada em diversos estudos como um fator mais confiável do que o Alfa de Cronbach $(\alpha)$ para efeitos de comparação. No entanto, nesta investigação os dois fatores (CC e $\alpha$ ) foram utilizados, além do índice Rho_A, que 
recentemente foi proposto na literatura como uma forma de estimar a confiabilidade por meio dos pesos fatoriais. Portanto, a consistência interna do modelo indica confiabilidade de coerência interna satisfatória (HAIR JR; RINGLE; SARSTEDT, 2013).

Realizou-se também a conferência da validação convergente do questionário, em que a variância média extraída (AVE) de cada dimensão se apresentou acima de 0,5 , atendendo o pressuposto de Hair Jr, Ringle, Sarstedt (2013), conforme Tabela 1.

Tabela 1 - Análise de mensuração inicial do modelo

\begin{tabular}{l|c|c|c|c|c|c}
\multicolumn{1}{c|}{ CONSTRUCTO } & $\boldsymbol{\beta}$ & $\boldsymbol{p}$ & $\boldsymbol{\alpha}$ & rho_A & CC & AVE \\
\hline APLICAÇÃO $\rightarrow$ ACAP & 0,228 & $<0,001$ & 0,941 & 0,945 & 0,958 & 0,851 \\
\hline AQUISIÇÃO $\rightarrow$ ACAP & 0,301 & $<0,001$ & 0,947 & 0,950 & 0,960 & 0,827 \\
\hline ASSIMILAÇÃO $\rightarrow$ ACAP & 0,251 & $<0,001$ & 0,894 & 0,904 & 0,923 & 0,707 \\
\hline TRANSFORMAÇÃO $\rightarrow$ ACAP & 0,298 & $<0,001$ & 0,910 & 0,920 & 0,931 & 0,693 \\
\hline ESTRUTURAL $\rightarrow$ CI & 0,455 & $<0,001$ & 0,930 & 0,932 & 0,942 & 0,642 \\
\hline HUMANO $\rightarrow$ CI & 0,303 & $<0,001$ & 0,873 & 0,933 & 0,891 & 0,509 \\
\hline RELACIONAL $\rightarrow$ CI & 0,355 & $<0,001$ & 0,884 & 0,908 & 0,908 & 0,530 \\
\hline DI $\rightarrow$ EFICÁCIA & 0,978 & $<0,001$ & 0,622 & 0,752 & 0,779 & 0,507 \\
\hline DI $\rightarrow$ EFICIÊNCIA & 0,741 & $<0,001$ & 0,897 & 0,900 & 0,916 & 0,522 \\
\hline
\end{tabular}

Fonte: Dados da pesquisa.

Após realizada a AFC utilizou-se da análise descritiva para compreender a tendência das respostas e dar subsídios para análises posteriores (Tabela 2). Também foi analisado o modelo de mensuração ajustado, conforme Figura 2.

\subsection{ANÁLISE DESCRITIVA E VALIDEZ DISCRIMINANTE}

Com o modelo ajustado após a AFC, realizou-se o cálculo da média de cada dimensão e do desvio padrão, a fim de nortear as respostas e subsidiar as futuras análises, conforme apresentado na Tabela 1.

Observa-se na Tabela 1 por meio da média do $\mathrm{Cl}$ Humano $(4,617)$, os respondentes consideram que o conhecimento intrínseco nas pessoas pertencentes à organização está relacionado à capacidade, habilidade, experiência, criatividade e a inovação dos funcionários, o que converge com o destacado por Bontis (1999).

Ao analisar o construto $\mathrm{Cl}$ Relacional, percebeu-se a maior média $(5,576)$. Isso indica que a relação entre as pessoas e entre as organizações além de serem contínuas, são tratadas como essenciais e fundamentais para construção de projetos inovadores (VAZ; SELIG; VIEGAS, 2019; IENCIU; MATIS, 2011). Detalhe, percebeu-se aderência com os achados de Vaz e Selig (2016), Stewart (1998) e Edvinsson e Malone (1998) quando os autores identificaram que o bom relacionamento com seus colaboradores, clientes e stakeholders, tornam-se o ativo intangível mais valioso da organização. Cassol et al. (2016) expõe que essa proximidade nos relacionamentos possibilita a melhoria e a inovação em processos, em produtos e na gestão organizacional.

Ao checar a Tabela 1, observa-se por meio dos respondentes que o $\mathrm{Cl}$ Estrutural se apresenta como ferramenta transformadora do conhecimento individual. $E$, ao verificar a média de 5,076, percebe-se a importância desta variável nas organizações, pois, é também por meio da infraestrutura, dos instrumentos gerenciais e dos sistemas de informação que acontece a inovação e o desenvolvimento de novos produtos e serviços (VAZ; SELIG; VIEGAS, 2019; IENCIU; MATIS, 2011). 
Tabela 2 - Validez Discriminante - Correlação de Pearson e a raiz quadrada da AVE das variáveis latentes dos constructos de primeira ordem

\begin{tabular}{l|c|c|c|c|c|c|c|c|c|c|c}
\hline $\begin{array}{c}\text { Validez } \\
\text { Discriminante } \\
\text { do modelo final }\end{array}$ & $\sim \mathbf{X}$ & $\mathbf{S}$ & $\begin{array}{c}\text { CA } \\
\text { Aplic. }\end{array}$ & $\begin{array}{c}\text { CA } \\
\text { Aqu. }\end{array}$ & $\begin{array}{c}\text { CA } \\
\text { Ass. }\end{array}$ & $\begin{array}{c}\text { CA } \\
\text { Tran. }\end{array}$ & Cl Est. & $\begin{array}{c}\text { CI } \\
\text { Hum. }\end{array}$ & Cl Rel. & $\begin{array}{c}\text { DI } \\
\text { Efici. }\end{array}$ & $\begin{array}{c}\text { DI } \\
\text { Eficá. }\end{array}$ \\
\hline CA Aplicação & $\mathbf{5 , 3 2 8}$ & $\mathbf{1 , 3 9 6}$ & $\mathbf{0 , 9 2 2}$ & & & & & & & & \\
\hline CA Aquisição & $\mathbf{5 , 1 4 6}$ & $\mathbf{1 , 5 3 4}$ & 0,839 & $\mathbf{0 , 9 0 4}$ & & & & & & & \\
\hline CA Assimilaç. & $\mathbf{4 , 9 2 9}$ & $\mathbf{1 , 5 6 5}$ & 0,827 & 0,821 & $\mathbf{0 , 8 4 1}$ & & & & & & \\
\hline CA Transfor. & $\mathbf{4 , 8 4 8}$ & $\mathbf{1 , 5 5 9}$ & 0,888 & 0,767 & 0,839 & $\mathbf{0 , 8 1 9}$ & & & & & \\
\hline Cl Estrutural & $\mathbf{5 , 0 7 6}$ & $\mathbf{1 , 4 8 1}$ & 0,805 & 0,791 & 0,786 & 0,814 & $\mathbf{0 , 8 3 5}$ & & & & \\
\hline Cl Humano & $\mathbf{4 , 6 1 7}$ & $\mathbf{1 , 5 7 4}$ & 0,649 & 0,618 & 0,626 & 0,706 & 0,685 & $\mathbf{0 , 8 4 9}$ & & & \\
\hline Cl Relacional & $\mathbf{5 , 5 7 6}$ & $\mathbf{1 , 2 1 2}$ & 0,784 & 0,842 & 0,768 & 0,698 & 0,768 & 0,546 & $\mathbf{0 , 8 8 3}$ & & \\
\hline DI Eficiência & $\mathbf{4 , 6 6 7}$ & $\mathbf{1 , 3 7 4}$ & 0,275 & 0,441 & 0,336 & 0,368 & 0,194 & 0,527 & 0,315 & $\mathbf{0 , 8 1 8}$ & \\
\hline DI Eficácia & $\mathbf{4 , 5 6 0}$ & $\mathbf{1 , 3 0 9}$ & 0,678 & 0,696 & 0,712 & 0,782 & 0,689 & 0,607 & 0,597 & 0,485 & $\mathbf{0 , 8 2 2}$ \\
\hline
\end{tabular}

Fonte: Elaborada pelos autores.

O constructo ACAP apresentou coerência entre os respondentes. Os quatro tipos de Capacidade Absortiva se apresentaram com médias semelhantes que variam de 4,868 (CA Transformação) a 5,328 (CA Aplicação). As percepções dos respondentes indicam que há nas indústrias ações e atividades que despertam a identificação e a aquisição do conhecimento até o momento de colocá-las em prática, ou seja, aplicá-las (ZAHRA; GEORGE, 2002).

Quanto a percepção relacionada às variáveis da dimensão Desempenho Inovador, percebeu-se que os respondentes possuem desempenho acima da média, uma vez que o resultado da variável Eficácia $(4,560)$ e da Eficiência $(4,667)$ apresentaram-se acima de 3,5. Quanto à Eficácia, isso é notado ao afirmarem que o nível de desempenho de sua empresa em comparação com a concorrência é superior em aspectos como redução de custos na produção, na qualidade do produto e em redução de danos ao meio ambiente. Isso converge com os entendimentos de Alegre, Lapiedra e Chiva (2006), Bakar e Ahmad (2010) e Henttonen, Ritala e Jauhiainen (2011) ao identificarem que a eficácia tem o objetivo de verificar como a inovação impacta de forma econômica na empresa, refletindo consequentemente, em seu nível de sucesso.

A Eficiência, por sua vez, expressada por Alegre, Lapiedra e Chiva (2006) e Brown e Eisenhardt (1995) como o esforço realizado para alcançar determinado resultado também é observada positivamente pelos respondentes. Dessa forma, os colaboradores pesquisados entendem que são mais eficientes que a concorrência no que tange o tempo utilizado para o desenvolvimento de projetos de inovação, no custo do projeto, bem como no grau de satisfação geral de todos envolvidos no projeto.

No tocante ao teste da Validade Discriminante (Tabela 2) Hair et al. (2005) indicam que ela verifica se a medida em questão não está relacionada indevidamente com indicadores de constructos distintos. Percebeu-se que ao utilizar o critério de Fornell-Larcker para a avaliação da validade, e, com o objetivo de comparar as raízes quadradas dos valores de AVE de cada constructo com as correlações entre as variáveis latentes dos constructos, verificouse que todos os valores de AVE são maiores que as demais correlações apresentadas, o que indica validade discriminante entre os constructos. 


\subsection{MODELO DE MENSURAÇÃO AJUSTADO}

Ao analisar o modelo após a análise fatorial confirmatória os testes apresentaram índices mais significativos que o modelo inicial. A Figura 2 e a Tabela 3 demonstram os dados após os ajustes.

Figura 2 - Modelo de mensuração ajustado

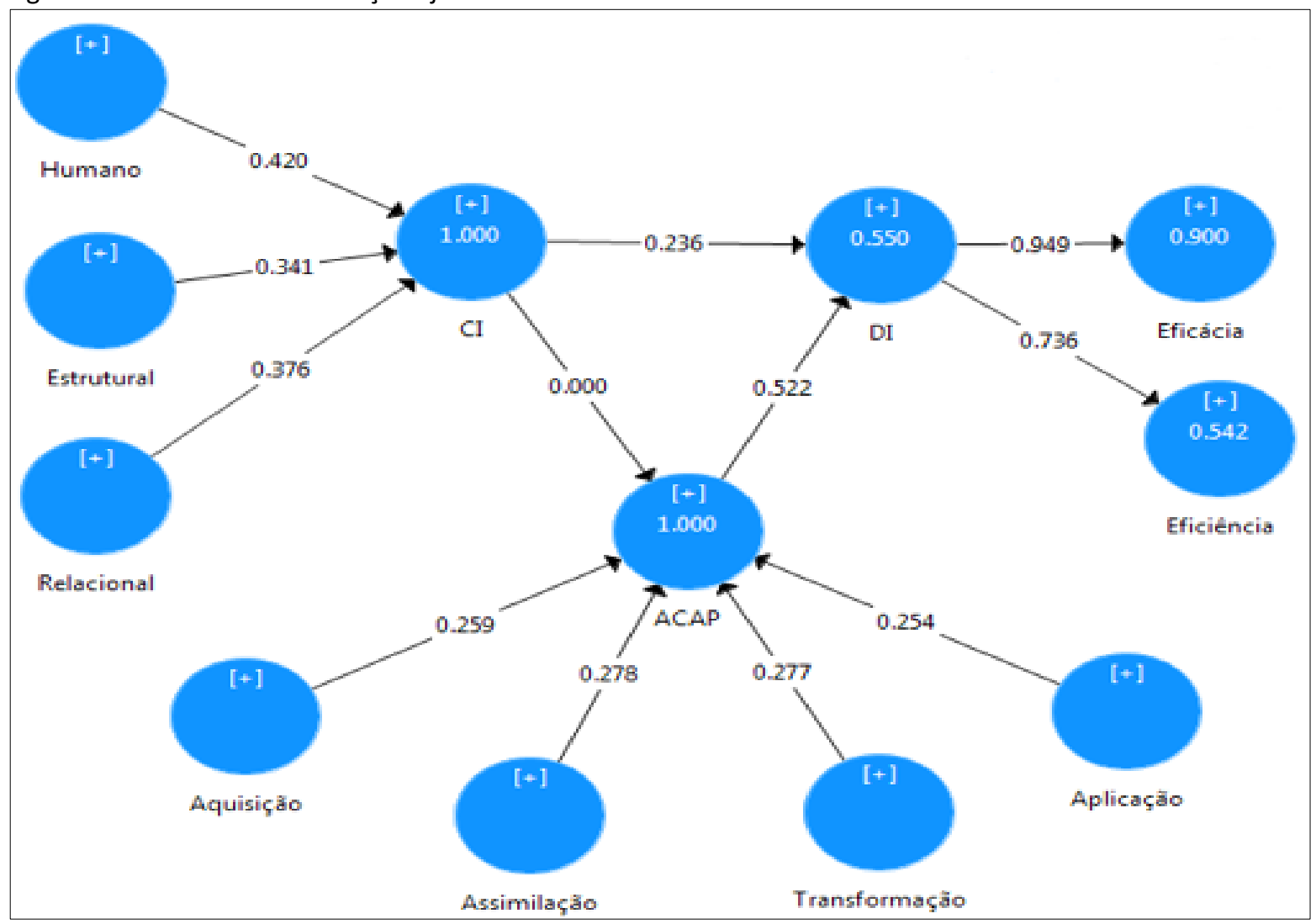

Fonte: Elaborado pelos autores.

A mensuração do modelo ajustado foi realizada em etapas. Na primeira, avaliou-se os coeficientes de Pearson $\left(R^{2}\right)$, que indicam a porção da variância das variáveis endógenas explicadas pelo modelo estrutural. Sugere-se que os valores de $R^{2}$ com limites em $2 \%, 13 \%$ e $26 \%$, podem ser considerados, respectivamente, como pequeno, médio ou grande efeito (COHEN, 1992). Percebe-se na Figura 2 que o valor de $R^{2}$ foi superior a $26 \%\left(R^{2} D I=0,550\right)$, indicando grande efeito do indicador para o modelo.

Tabela 3 - Modelo de Mensuração Ajustado

\begin{tabular}{l|c|c|c|c|c|c}
\multicolumn{1}{c|}{ CONSTRUCTO } & B & $\boldsymbol{p}$ & $\boldsymbol{\alpha}$ & rho_A & CC & AVE \\
\hline APLICAÇÃO $\rightarrow$ ACAP & 0,254 & 0,000 & 0,941 & 0,945 & 0,958 & 0,851 \\
\hline AQUISIÇÃO $\rightarrow$ ACAP & 0,259 & 0,000 & 0,925 & 0,928 & 0,947 & 0,817 \\
\hline ASSIMILAÇÃO $\rightarrow$ ACAP & 0,278 & 0,000 & 0,894 & 0,904 & 0,923 & 0,707 \\
\hline TRANSFORMAÇÃO $\rightarrow$ ACAP & 0,277 & 0,000 & 0,876 & 0,889 & 0,910 & 0,670 \\
\hline ESTRUTURAL $\rightarrow$ CI & 0,341 & 0,000 & 0,856 & 0,862 & 0,962 & 0,697 \\
\hline HUMANO $\rightarrow$ CI & 0,420 & 0,000 & 0,901 & 0,920 & 0,927 & 0,720 \\
\hline RELACIONAL $\rightarrow$ CI & 0,376 & 0,000 & 0,905 & 0,908 & 0,934 & 0,781 \\
\hline DI $\rightarrow$ EFICÁCIA & 0,949 & 0,000 & 0,879 & 0,883 & 0,912 & 0,675 \\
\hline DI $\rightarrow$ EFICIÊNCIA & 0,736 & 0,000 & 0,758 & 0,775 & 0,858 & 0,669 \\
\hline FOnte: EIabOra
\end{tabular}

Fonte: Elaborada pelos autores 
Verificou-se que os coeficientes de caminho do modelo estrutural, os quais também podem ser interpretados como coeficientes beta ( $\beta$ - coeficientes de regressão padronizados) de mínimos quadrados, em sua maioria, superaram o modelo anterior. Dessa forma o modelo de mensuração ficou ajustado. A partir de então, a Tabela 4 demonstra os resultados das hipóteses testadas.

Tabela 4 - Resultados das hipóteses testadas

\begin{tabular}{l|l|c|c|c}
\hline Hipóteses & Caminho estrutural & $\boldsymbol{\beta}$ & $\boldsymbol{p}$-value & Resultado \\
\hline $\mathrm{H} 1$ & $\mathrm{Cl} \rightarrow \mathrm{DI}$ & 0,236 & 0,000 & Suportada \\
\hline $\mathrm{H} 2$ & $\mathrm{Cl} \rightarrow \mathrm{ACAP}$ & 0,000 & 0,774 & Não Suportada \\
\hline $\mathrm{H} 3$ & $\mathrm{ACAP} \rightarrow \mathrm{DI}$ & 0,522 & 0,000 & Suportada \\
\hline $\mathrm{H} 4$ & $\mathrm{Cl} \rightarrow \mathrm{ACAP} \rightarrow \mathrm{DI}$ & 0,000 & 0,025 & Não Suportada \\
\hline
\end{tabular}

Fonte: Elaborada pelos autores.

De acordo com a Figura 2 o Cl possui influência de 0,236 sobre o DI. Esta influência é validada pelo resultado da carga $\beta$, dando suporte para confirmar a hipótese $H 1$, onde o capital intelectual influencia positivamente no desempenho inovador. Esse resultado corrobora com a visão de Gracioli et al. (2012) uma vez que o Cl é responsável por uma parcela cada vez maior no desempenho das organizações. Os resultados também indicam que as organizações se importam com o desenvolvimento do colaborador, para que ele, por meio de suas ideias, inicie e instrumente produtos e processos inovadores, constantemente. Nesse sentido, pode-se ainda destacar a eficiência e a eficácia das indústrias pesquisadas, mediante ao observado nas cargas $\beta$ de 0,949 para Eficácia e 0,736 para Eficiência.

A influência do $\mathrm{Cl}$ para a ACAP apresentou-se fraca $(0,000)$, não obteve significância $(P$-value $=0,774)$, rejeitando a $\mathrm{H} 2$, ao testar se o capital intelectual influencia positivamente na capacidade absortiva. Esse resultado se mostrou diferente na amostra estudada e encontrada em pesquisas anteriores ao sugerirem que o processo de gestão do $\mathrm{Cl}$ está relacionado positivamente com o desenvolvimento da ACAP da organização (NAZARPOORI, 2017; CASSOL et. al., 2016).

Ao testar se a capacidade absortiva influencia positivamente no desempenho inovador (H3), percebeu-se que há relação. A carga fatorial demonstrou influência de 0,522 com significância de 0,000 ( $P$-value). Dessa forma, os testes suportam a hipótese 3 , visto que as organizações que possuem a capacidade de adquirir, assimilar, transformar e aplicar conhecimento, também conseguem ter maior vantagem competitiva (NAZARPOORI, 2017; CASSOL et. al., 2016). Além disso, os resultados indicam que as organizações estão atentas aos acontecimentos externos, buscando absorver e utilizar diversos mecanismos com o objetivo de diminuir as barreiras organizacionais e aumentar a competitividade. Infere-se ainda que há esforços organizacionais quanto a ampliação da base de conhecimento, alavancagem de novos processos, como também o desenvolvimento de novos recursos estratégicos industriais.

A hipótese 4 que testa se a capacidade absortiva media a relação entre capital intelectual e desempenho inovador, apresenta-se com influência de 0,000 (multiplicação de $0,000 \times 0,522$ ). Sendo assim, rejeita-se a H4, uma vez que não se encontrou efeito mediador da ACAP entre o $\mathrm{Cl}$ e o DI. 


\section{CONSIDERAÇÕES FINAIS}

O ambiente de mercado, de crescente competição das indústrias brasileiras, se mostra cada vez mais complexo e multifacetado. Na medida em que as organizações diversificam a oferta de produtos e serviços, os consumidores constituem nichos específicos, que exigem, na maioria das vezes, a concepção de soluções customizadas, sem custos adicionais.

A gestão do capital intelectual e da capacidade absortiva são considerados recursos importantes para que uma organização desenvolva vantagem competitiva. No contexto pesquisado, a vantagem competitiva refere-se ao desempenho inovador constituído por eficiência e eficácia em produtos e processos.

A investigação teve como objetivo analisar a gestão do capital intelectual e da capacidade absortiva no desempenho inovador. Utilizou-se da abordagem descritiva, confirmatória e quantitativa como metodologia na abordagem de 97 indústrias do segmento metalmecânico.

Percebeu-se por meio da análise de dados descritiva que o desempenho inovador apresenta-se como positivo nas organizações. Nesse contexto, notou-se a capacidade que a empresa possui de minimizar recursos em suas atividades, identificar oportunidades, melhorando a qualidade dos produtos frente a concorrência (eficácia). Além disso, observase por meio da eficiência que seus esforços superam da concorrência, objetivando menor tempo no desenvolvimento dos projetos de inovação e menor custo.

Em relação aos estudos idealizados pela Modelagem de Equações Estruturais observa-se que há influência positiva do $\mathrm{Cl}$ sobre o DI, bem como influência do ACAP no DI. As hipóteses suportadas corroboraram com estudos anteriores envolvendo capital intelectual e capacidade absortiva e a influência positiva de ambos sobre o desempenho (CASSOL et al., 2016; GRACIOLI et al., 2012). Já as hipóteses rejeitadas se mostraram diferentes na amostra estudada, pois não foi encontrado influência do capital intelectual sobre a capacidade absortiva (NAZARPOORI, 2017; CASSOL et al., 2016), bem como também não houve o efeito mediador esperado da capacidade absortiva.

Portanto, entende-se que a ACAP e o $\mathrm{Cl}$ possuem atributos que influenciam isoladamente no DI das organizações em questão. Além disso, observou-se que as pessoas utilizam de todos os mecanismos disponíveis para suprir a demanda exposta no mercado, seja relacional, humano ou estrutural.

Tem-se como limitação de pesquisa um único segmento industrial pesquisado, bem como a indagação de apenas um integrante por indústria, o que oportuniza a realização de futuros trabalhos com vários profissionais de cada indústria e entre segmentos, comparandoos e relacionando-os. Possibilita-se acrescentar novas variáveis ao modelo utilizado, incluindo o desempenho e o comprometimento organizacional entre a ACAP e o $\mathrm{CI}$.

\section{REFERÊNCIAS}

ALEGRE, J.; LAPIEDRA, R.; CHIVA, R. A measurement scale for product innovation performance. European Journal of Innovation Management, Melbourne, v. 9, n. 4, p. 333346, out. 2006. DOI: https://doi.org/10.1108/14601060610707812.

ALEGRE, J.; CHIVA, R. Assessing the impact of organizational learning capability on product innovation performance: an empirical test. Technovation, v. 28, n. 1, p. 315-326, jun. 2008. DOI: https://doi.org/10.1016/j.technovation.2007.09.003. 
ALEGRE, J; CHIVA, R. Linking entrepreneurial orientation and firm performance: The role of organizational learning capability and innovation performance. Journal of Small Business Management, v. 51, n. 4, p. 491-507, jan. 2013. DOI: https://doi.org/10.1111/jsbm.12005.

BAKAR, L. J. A.; AHMAD, H. Assessing the relationship between firm resources and product innovation performance: A resource-based view. Business Process Management Journal, v. 16, n. 3, p. 420-435, jun. 2010. DOI: https://doi.org/10.1108/14637151011049430

BECKER, G. S. Investment in human capital: A theoretical analysis. Journal of Political Economy, New York, v. 70, n. 5, p. 9-49, out. 1962. DOI: https://doi.org/10.1086/258724

BONTIS, N. Managing organizational knowledge by diagnosing intellectual capital: framing and advancing the state of the field. International Journal of Technology Management, $\mathrm{v}$. 18, n. 5-8, p. 433-462, 1999. DOI: https://doi.org/10.1504/IJTM.1999.002780.

BRANCO, M.; DELGADO, C. Research on corporate social responsibility and disclosure in Portugal. Social Responsibility Journal, v. 7, n. 2, p. 202-217, jun. 2011. DOI:

https://doi.org/10.1108/17471111111141495.

BROWN, S. L.; EISENHARDT, K. M. Product development: past research, present, findings, and future directions. The Academy of Management Review, Westchester, v. 20, n. 2, p. 343.378, abr. 1995. DOI: https://doi.org/10.2307/258850.

CABRITA, M. Capital Intelectual e desempenho organizacional. Lisboa: Lidel, 2009.

CABRITA, M.; BONTIS, N. Intellectual Capital and business performance in the Portuguese banking industry, International Journal Technology Management, v. 43, p. 212-237. jul. 2008. DOI: https://doi.org/10.1504/IJTM.2008.019416.

CARSON, E.; RANZIJN, R.; WINEFIELD, A; MARSDEN, H. Intellectual Capital: Mapping Employee and Work Group Attributes. Journal of Intellectual Capital. v.5, p.443-463, set. 2004. DOI: https://doi.org/10.1108/14691930410550390.

CASSOL, A.; REIS GONÇALO, C.; SANTOS, A.; LIMA RUAS, R. A administração estratégica do capital intelectual: Um modelo baseado na capacidade absortiva para potencializar a inovação. Revista Ibero-Americana de Estratégica - RIAE. São Paulo, v. 15, n. 1, jan./mar. 2016. DOI: https://doi.org/10.5585/riae.v15i1.2161.

CHEN, Y.; WANG, Y.; NEVO, S.; BENITEZ-AMADO, J.; KOU, G. IT capabilities and product innovation performance: The roles of corporate entrepreneurship and competitive intensity. Information \& Management, v. 52, n. 6, p. 643-657, set. 2015. DOI: https://doi.org/10.1016/j.im.2015.05.003.

CHIN, W. W.; NEWSTED, P. R. Structural equation modeling analysis with small samples using partials least squares. In: HOYLE, R. H. Statistical Strategies for Small Sample Research, Sage, 1999. 
CHIVA, R.; ALEGRE, J. Organizational Learning and Organizational Knowledge Towards the Integration of Two Approaches. Management Learning, London, v. 36, n.1, p. 49-68, mar. 2005. DOI: https://doi.org/10.1177/1350507605049906.

COHEN, J. A power primer. Psychological Bulletin, New York, v. 112, n. 1, p. 155-159, jul. 1992. DOI: https://doi.org/10.1037/0033-2909.112.1.155.

COHEN, W. M.; LEVINTHAL, D. A. Innovation and learning: the two faces of R\&D. The Economic Journal, Oxford, v. 99, n. 1, p 569-596, set. 1989. DOI:

https://doi.org/10.2307/2233763.

COHEN, W.; LEVINTHAL, D. Absorptive capacity: a new perspective on learning and innovation. Administrative Science Quarterly, New York, v. 35, n. 1, p. 128-152, mar. 1990. DOI: https://doi.org/10.2307/2393553.

CRICELLI, L.; GREGO, M.; GRIMALDI, M. An overall index of intellectual capital. Management Research Review, v. 37, n. 10, p. 541-552, set. 2014. DOI: https://doi.org/10.1108/MRR-042013-0088.

CURADO, C.; OLIVEIRA, M.; MAÇADA, A. C. G.; NODARI, F. Teams' innovation: getting there through knowledge sharing and absorptive capacity. Knowledge Management Research \& Practice, v. 15, n. 1, p. 45-53, dez. 2017. DOI: https://doi.org/10.1057/kmrp.2015.8.

DAVENPORT, T. H.; PRUSAK, L. Working Knowledge: how organizations manage what they know. New York: Harvard Business School Press, 1998.

EDVINSSON, L.; MALONE, M. Capital Intelectual. São Paulo: Makron Books, 1998.

EASTERBY-SMITH, M. Disciplines of organizational learning: Contributions and Critiques. Human Relations, Lancaster, v. 50, n. 9, p. 1085-1113, set. 1997. DOI: https://doi.org/10.1177/001872679705000903.

FERREIRA, G. C.; FERREIRA, J. J. Absorptive capacity: an analysis in the context of brazilian family firms. RAM-Revista de Administração Mackenzie, São Paulo, v. 18, n. 1, p. 174-204, jan/fev. 2017. DOI: http://dx.doi.org/10.1590/1678-69712017/administracao.v18n1p174-204.

FIESC. Federação das Indústrias do Estado de Santa Catarina - Setores portadores de futuro para a indústria catarinense 2022. Florianópolis. 2016. Retrieved from http://www4.fiescnet.com.br/images/banner-pedic/documento-oficial-setores.pdf <acesso em: 21 jan. 2019>.

GRACIOLI, C.; GODOY, L. P.; LORENZETT, D. P.; GODOY, T. P. Capital Intelectual: Uma ferramenta inovadora na busca por vantagens competitivas. Revista de Administração e Inovação, São Paulo, v. 9, n. 4, p. 96-120, out./dez. 2012. DOI: https://doi.org/10.5773/rai.v9i4.778.

GUNDAY, G.; ULUSOY, G.; KILIC, K.; ALPKAN, L. Effects of innovation types on firm performance. International Journal of Production Economics, Amsterdam, v. 133, n. 2, p. 662-676, out. 2011. DOI: https://doi.org/10.1016/j.ijpe.2011.05.014. 
HAIR JR, J. F.; BABIN, B.; MONEY, A; SAMOUEL, P. Fundamentos de métodos de pesquisa em administração. Porto Alegre: Bookman, 2005.

HAIR, JR. F.; RINGLE, C. M.; SARSTEDT, M. Partial least squares structural equation modeling: Rigorous applications, better results and higher acceptance. Long Range Planning, v. 46, n. 1-2, p. 1-12, mar. 2013. DOI: https://doi.org/10.1016/j.Irp.2013.01.001.

HENTTONEN, K.; RITALA, P.; JAUHIAINEN, T. Exploring open search strategies and their perceived impact on innovation performance - empirical evidence. International Journal of Innovation Management, London, v. 15, n. 3, p. 525-541, jun. 2011. DOI:

https://doi.org/10.1142/S1363919611003428.

HUNG, H. Influence of the environment on innovation of performance of TQM. Total Quality Management \& Business Excellence, Abingdon, v. 18, n. 7, p. 715- 730, set. 2007. DOI: https://doi.org/10.1080/14783360701349666.

IENCIU, N. M.; MATIS, D. A theoretical framework of intellectual capital. International Journal of Business Research, v. 11, n. 2, p. 131-136, mar. 2011.

JIMÉNEZ-JIMÉNEZ, D.; SANZ-VALLE, R. Innovation, organizational learning, and performance. Journal of Business Research, v. 64, n. 4, p. 408-417, abr. 2011. DOI: https://doi.org/10.1016/j.jbusres.2010.09.010.

JONASH, R. S.; SOMMERLATTE, T. O valor da inovação: como as empresas mais avançadas atingem alto desempenho e lucratividade. Rio de Janeiro: Campus, 2001.

JORDÃO, R. V. D.; NOVAS, J. C. Knowledge management and intellectual capital in networks of small and médium-sized enterprises. Journal of Intellectual Capital. v. 18, n. 3, p. 1-27, jul. 2017. DOI: https://doi.org/10.1108/JIC-11-2016-0120.

KAPLAN, R. S.; NORTON, D. P. A estratégia em Ação. Rio de Janeiro: Campus, 1997.

KIANTO, A.; SÁENZ, J.; ARAMBURU, N. Knowledge-based human resource management practices, intellectual capital and innovation. Journal of Business Research, v. 81, p. 11-20, dez. 2017. DOI: https://doi.org/10.1016/j.jbusres.2017.07.018

KIM, N.; SHIM, C. Social capital, knowledge sharing and innovation of small-and mediumsized enterprises in a tourism cluster. International journal of contemporary hospitality management, v. 30 n. 6, p. 2417-2437, jun. 2018. DOI: https://doi.org/10.1108/IJCHM-07-20160392

LIAO, S.; CHANG, W.; WU, C. Exploring TQM-Innovation relationship in continuing education: a system architecture and propositions. Total Quality Management \& Business Excellence, Abingdon, v. 21, n. 11, p. 1121-1139, nov. 2010. DOI:

https://doi.org/10.1080/14783363.2010.529330.

MARIANO, S., WALTER, C. The construct of absorptive capacity in knowledge management and intellectual capital research: content and text analyses. Journal of Knowledge Management, v. 19, n. 2, p. 372-400, abr. 2015. DOI: https://doi.org/10.1108/JKM-08-20140342. 
MCPHAIL, K. Where is the ethical knowledge in the knowledge economy? Power and potential in the emergence of ethical knowledge as a component of intellectual capital. Critical Perspectives on Accounting, v. 20, n. 7, p. 804-822, out. 2009. DOI: https://doi.org/10.1016/j.cpa.2008.09.004.

MORÉ, R. P. O.; GONÇALO, C. R.; VARGAS, S. M. L.; BUCIOR, E. R.; CEMBRANEL, P. Capacidade absortiva no contexto da inovação: um estudo bibliométrico. Desenvolve Revista de Gestão do Unilasalle, Canoas, v. 3, n. 1, p.113-126, mar. 2014. DOI: http://dx.doi.org/10.18316/1215.

MORENO, P. I.; REALY, J. C. M.; ROSA, D. La incidencia del capital humano y la cultura emprendedora en la innovación. Cuadernos de Economía y Dirección de la Empresa, v. 14, n. 2, p. 139-150, jul/set. 2011. DOI: https://doi.org/10.1016/j.cede.2010.09.001.

NAHAPIET, J.; GHOSHAL, S. Social capital, intellectual capital, and the organizational advantage. The Academy of Management Review, Westchester, v. 23, n. 2, p. 242-266, abr. 1998. DOI: https://doi.org/10.5465/amr.1998.533225.

NAZARPOORI, A. H. Survey the effects of intellectual capital and absorptive capacity on innovation capability (Case Study of Saipa Company in Tehran). International Journal of Innovation Management, v. 21, n. 03, p. 1-19, abr. 2017. DOI: https://doi.org/10.1142/S1363919617500293.

ROSENBUSCH, N.; BRINCKMANN, J.; BAUSCH, A. Is innovation always beneficial? A metaanalysis of the relationship between innovation and performance in SMEs. Journal of business Venturing, v. 26, n. 4, p. 441-457, jul. 2011. DOI:

https://doi.org/10.1016/j.jbusvent.2009.12.002.

SOO, C.; TIAN, A. W.; TEO, S. T.; CORDERY, J. Intellectual capital-enhancing HR, absorptive capacity, and innovation. Human resource management, v. 56, n. 3, p. 431-454, maio/jun. 2017. DOI: https://doi.org/10.1002/hrm.21783.

STEWART, T. A. Capital Intelectual - A nova vantagem competitiva das empresas. Rio de Janeiro: Campus Ltda., 1998.

SUBRAMANIAM, M.; YOUNDT, M. A. The influence of intellectual capital on the types of innovative capabilities. The Academy of Management Journal, Westchester, v. 48, n. 3, p. 450-463, jul. 2005. DOI: https://doi.org/10.5465/amj.2005.17407911.

SVEIBY, K. E. The new organizational wealth: Managing \& measuring knowledge-based assets. New York: Berrett-Koehler Publishers, 1997.

SVEIBY, K. E. A nova riqueza das organizações: gerenciando e avaliando patrimônios de conhecimento. Rio de Janeiro: Campus, 1998.

TEECE, D. J.; PISANO, G.; SHUEN, A. Dynamic capabilities and strategic management. Strategic Management Journal, New York, v. 18, n. 7, p. 509-533, mar. 1997. DOI: https://doi.org/10.1002/(SICI)1097-0266(199708)18:7<509::AID-SMJ882>3.0.CO;2-Z. 
TODOROVA, G.; DURISIN, B. Absorptive Capacity: valuing a reconceptualization. The Academy of Management Review, Westchester, v. 32, n. 3, p. 774-786, jul. 2007. DOI: https://doi.org/10.5465/amr.2007.25275513.

VAZ, C. R.; SELIG, P. M. Maturity Assessment Model of Intellectual Capital for Manufacturing Organization. IEEE Latin America Transactions, v. 14, n. 1, p. 206-219, mar. 2016. DOI: https://doi.org/10.1109/TLA.2016.7430081.

VAZ, C. R.; SELIG, P. M.; VIEGAS, C. V. A proposal of intellectual capital maturity model (ICMM) evaluation. Journal of Intellectual Capital, v. 20, n. 2, p. 208-234, abr. 2019. DOI: https://doi.org/10.1108/JIC-12-2016-0130.

WHEELWRIGHT, S.C.; CLARK, K.B. Revolutionizing Product Development - Quantum Leaps in Speed, Efficiency, and Quality, The Free Press, New York, NY, 1992.

YOUNDT, M. A.; SUBRAMANIAM, M.; SNELL, S. A. Intellectual capital profiles: $\mathrm{Na}$ examination of investments and returns. Journal of Management Studies, Oxford, v. 41, n. 2, p. 335-362, fev. 2004. DOI: https://doi.org/10.1111/j.1467-6486.2004.00435.x.

ZAHRA, S. A.; GEORGE, G. Absorptive capacity: a review, reconceptualization, and extension. The Academy of Management Review, Westchester, v. 2, n. 17, p. 185-203, abr. 2002. DOI: https://doi.org/10.5465/amr.2002.6587995.

ZHUANG, L.; WILIAMSON, D.; CARTER, M. Innovate or liquidate - are all organizations convinced? A two-phased study into the innovation process. Management Decision, v. 37, $\mathrm{n}$. 1, p. 57-71, fev. 1999. DOI: https://doi.org/10.1108/00251749910252030.

ZOLLO, M.; WINTER, S. G. Deliberate learning and the evolution of dynamic capabilities. Organization Science, Pennsylvania, v. 13, n. 3, p. 339-351, jul. 2002. DOI: https://doi.org/10.1287/orsc.13.3.339.2780. 